\title{
ALIMENTOS FONTES DE FERRO E VITAMINA C CONSUMIDOS ENTRE LACTENTES DA ATENÇÃO PRIMÁRIA À SAÚDE
}

\author{
Luana Lemos Leão ${ }^{1}$ Barbara Andrade Antunes ${ }^{1}$, Carolina Amaral Oliveira², Maria Fernanda Santos \\ Figueiredo Brito ${ }^{3}$, Lucinéia de Pinho ${ }^{4}$.
}

RESUMO: Objetivo: avaliar o consumo de alimentos fontes de ferro e vitamina $C$ entre crianças de 0 a 24 meses. Métodos: trata-se de estudo transversal realizado com 357 crianças durante campanha de vacinação em novembro de 2014 em Montes Claros, MG. O consumo de alimentos fontes de ferro e vitamina C foi avaliado com questionário de frequência alimentar. Realizou-se análise descritiva simples. Resultados: a amamentação foi verificada em 140 (93\%) crianças menores de seis meses. Na alimentação complementar entre os alimentos fontes de ferro, o consumo de carne cozida/moída foi frequente em 80 (38,2\%) e de vísceras foi baixo ou ausente em 147 (70,6\%). Todas as crianças consumiam feijão moderadamente ou frequentemente. O consumo frequente de laranja foi relatado em 74 (35,5\%). Conclusão: o consumo de alimentos fontes de ferro e vitamina C das crianças na Atenção Primária à Saúde é de baixa frequência, apresentando risco para anemia ferropriva.

DESCRITORES: Anemia ferropriva; Consumo alimentar; Atenção primária à saúde; lactente.

\section{FOOD SOURCES OF IRON AND VITAMIN C CONSUMED AMONG INFANTS IN PRIMARY HEALTHCARE}

ABSTRACT: Objective: to evaluate the consumption of food sources of iron and vitamin C among children from 0 to 24 months. Methods: This cross-sectional study was carried out with 357 children during the vaccination campaign in November 2014 in Montes Claros, Minas Gerais, Brazil. The consumption of food sources of iron and vitamin $C$ was evaluated using a food frequency questionnaire. A simple descriptive analysis was performed. Results: Breastfeeding was verified in $140(93 \%)$ children younger than 6 months. Regarding the supplementary feeding of iron source foods, the consumption of cooked/ground meat was frequent in $80(38.2 \%)$ children and the consumption of offal was low or absent in $147(70.6 \%)$. All the children moderately or frequently consumed beans. Frequent consumption of oranges was reported in 74 (35.5\%) children. Conclusion: The consumption of food sources of iron and vitamin C by children in Primary Healthcare presented a low-frequency, with a risk for iron deficiency anemia.

KEYWORDS: Iron deficiency anemia; Food consumption; Primary Healthcare; Infant.

\section{ALIMENTOS QUE SON FUENTES DE HIERRO Y VITAMINA C CONSUMIDOS ENTRE LACTENTES DE LA ATENCIÓN BÁSICA A LA SALUD}

RESUMEN: Objetivo: evaluar el consumo de alimentos que son fuentes de hierro y vitamina $\mathrm{C}$ entre niños de 0 a 24 meses. Métodos: estudio transversal realizado con 357 niños durante campaña de vacunación en noviembre de 2014 en Montes Claros, MG. Se evaluó el consumo de alimentos ricos en hierro y vitamina C por medio de cuestionario de frecuencia alimentar. Se realizó análisis descriptivo simple. Resultados: se verificó amamantamiento en 140 (93\%) niños con menos de seis meses. En la alimentación complementar entre los alimentos ricos en hierro, el consumo de carne cocida/molida fue frecuente en 80 (38,2\%) y de vísceras fue bajo o inexistente en 147 (70,6\%). Todos los niños consumían frijol de forma moderada o frecuente. El consumo a menudo de naranja fue apuntado en 74 (35,5\%). Conclusión: el consumo de alimentos ricos en hierro y vitamina C de los niños en la Atención Básica a la Salud tiene baja frecuencia y presenta riesgo para anemia ferropriva.

DESCRITORES: Anemia ferropriva; Consumo alimentar; Atención básica a la salud; lactente.

${ }^{1}$ Discente de Nutrição. Curso de Nutrição da Faculdade de Saúde Ibituruna. Montes Claros, MG, Brasil.

${ }^{2}$ Discente de Enfermagem. Curso de Enfermagem da Universidade Estadual de Montes Claros. Montes Claros, MG, Brasil.

${ }^{3}$ Enfermeira. Doutora em Ciências da Saúde. Docente da Faculdades Integradas Pitágoras e do Programa de Pós-graduação em Cuidado Primário em Saúde da Universidade Estadual de Montes Claros. Montes Claros, MG, Brasil.

${ }^{4}$ Nutricionista. Doutora em Ciências da Saúde. Docente da Faculdades Integradas Pitágoras e do Programa de Pós-graduação em Cuidado Primário em Saúde da Universidade Estadual de Montes Claros. Montes Claros, MG, Brasil. 


\section{INTRODUÇÃO}

A alimentação tem importante papel no processo de crescimento e desenvolvimento infantil ${ }^{(1,2,3)}$. O hábito alimentar é formado nos primeiros anos de vida e pode impactar nas condições de saúde ao longo da vida ${ }^{(4)}$. Inadequações no consumo alimentar podem resultar na gênese dos principais agravos nutricionais de saúde pública durante a infância ${ }^{(2)}$.

A ingestão de micronutrientes pelas crianças tem se mostrado inadequada, sobretudo em relação ao ferro, no cenário internacional ${ }^{(5-8)}$ e nacional ${ }^{(2,4)}$. Este nutriente é considerado como marcador de uma dieta de baixa qualidade e de práticas alimentares incorretas ${ }^{(2,4)}$.

A deficiência de ferro consiste na quantidade insuficiente desse nutriente no organismo para a manutenção das funções fisiológicas normais. Resulta de uma absorção inadequada desse mineral e de seus facilitadores de absorção, como a Vitamina $C^{(9)}$.

A anemia ferropriva, patologia resultante da carência de ferro, tem atingido muitos lactentes ${ }^{(1,5-7,10,11)}$. Nessa fase, a anemia está comumente relacionada com a interrupção precoce do aleitamento materno e a alimentação complementar oferecida de forma inadequada ${ }^{(3,10,12)}$. Atinge, principalmente, populações de países em desenvolvimento, com restrição ao acesso a uma nutrição equilibrada, a serviços de saúde e a sistemas de saneamento básico ${ }^{(10)}$.

As implicações desse quadro vão desde o comprometimento do desenvolvimento psicomotor, aumento da suscetibilidade a infecções, diminuição na capacidade de se exercitar e danos irreversíveis no sistema nervoso ${ }^{(1,5,9,10)}$. com repercussões na idade adulta. Esse quadro pode gerar consequências sociais e econômicas na sociedade $\mathrm{e}^{(1,9,10)}$.

Embora a anemia seja amplamente discutida pela comunidade científica, ainda persistem elevadas prevalências desse agravo na população brasileira, com peculiaridades regionais e de faixa etária ${ }^{(10)}$. A avaliação do consumo alimentar infantil constitui estratégia imprescindível para a prevenção da anemia ferropriva e da promoção em saúde, principalmente em populações de risco, ${ }^{(13)}$ como os lactentes acompanhados na Atenção Primária à Saúde (APS). Nesse sentido, o presente estudo tem como objetivo avaliar o consumo de alimentos naturais fontes de ferro e vitamina $C$ entre crianças de 0 a 24 meses.

\section{- MÉTODO}

Trata-se de uma pesquisa transversal, com abordagem quantitativa, realizada com crianças menores de 24 meses na campanha de vacinação em novembro de 2014 no município de Montes Claros, MG.

A população deste estudo foi composta pelas crianças nas faixas etárias de zero a 24 meses vacinadas durante a campanha de vacinação realizada na APS. O tamanho da amostra foi calculado considerando a prevalência de 24\% para anemia na infância, com intervalo de 95\% de confiança e nível de precisão de $5 \%$. Foi adotado um fator de correção para o efeito de desenho (deff) igual a 1,2 e uma taxa de não resposta de 10\%. Estimou-se a participação de 357 crianças no estudo. As unidades amostrais foram eleitas utilizando-se a amostragem probabilística por conglomerado em dois estágios. No primeiro estágio, foram selecionadas as Unidades Básicas de Saúde (UBS) e no segundo estágio as crianças em cada UBS, de forma sistemática.

A coleta de dados foi realizada por profissionais e acadêmicos da área de nutrição e enfermagem, devidamente capacitados com os pais ou responsáveis das crianças menores de 24 meses em agosto de 2014. Para tanto, utilizou-se um instrumento padronizado que contemplava as variáveis sociodemográficas (sexo, idade, escolaridade da mãe, tipo de residência e participação em programas sociais). Para a avaliação da alimentação infantil foi usado o formulário de marcadores do consumo alimentar adotado pelo Sistema de Vigilância Alimentar e Nutricional (SISVAN), recomendado pelo Ministério da Saúde ${ }^{(14)}$. Investigou-se questões relativas ao aleitamento materno, à introdução da alimentação complementar e à ingestão alimentar do dia anterior. 
O consumo de alimentos fontes de ferro e vitamina C foi avaliado por meio de um Questionário de Frequência Alimentar (QFA), composto por alimentos fontes desses nutrientes. O QFA contemplou a quantidade e a frequência de alimentos fontes de ferro e vitamina $C$ consumidos. A frequência alimentar foi classificada como nunca consumido, baixo consumo (menos de uma vez/mês a três vezes/mês); consumo moderado (uma vez/semana a quatro vezes/semana) e consumo frequente (uma vez/dia a duas ou mais vezes/dia).

Foi realizada a análise descritiva dos dados, por meio da frequência absoluta e relativa. No processamento estatístico, foi utilizado o software estatístico Statistical Package for the Social Sciences $\left(\mathrm{SPSS}^{\circledR}\right)$, versão 19.0 para Windows.

Este estudo foi aprovado pelo Comitê de Ética em Pesquisa (CEP) n.. 418.216/13 das Faculdades Unidas do Norte de Minas - FUNORTE. Todos os participantes da pesquisa assinaram um Termo de Consentimento Livre e Esclarecido.

\section{RESULTADO}

Participaram do estudo 357 crianças entre 0 e 24 meses, sendo 202 (57\%) do sexo feminino; com idade entre 6 e 24 meses, 207 (58\%); e pertencentes à classe econômica C, 167 (47\%). Quanto às características das mães, aproximadamente metade da amostra, 170 (48\%), tinha idade inferior a 30 anos; 184 (52\%) possuíam apenas um filho; e 188 (53\%) participavam de programas sociais do governo. Com relação à escolaridade, 200 (56\%) mães possuíam oito anos ou menos de estudo (Tabela 01).

Tabela 1 - Distribuição de crianças, segundo características sociodemográficas. Montes Claros, MG, Brasil, 2014. (continua)

\begin{tabular}{lcc}
\hline Variáveis & N & \% \\
\hline Sexo da criança & 155 & 43 \\
Masculino & 202 & 57 \\
Feminino & & \\
\hline Idade Materna (anos) & 170 & 48 \\
$\leq 30$ & 133 & 37 \\
30 a 40 & 54 & 15 \\
40 a 50 & & \\
\hline Idade da criança (meses) & 15 & 04 \\
$<1$ mês & 135 & 38 \\
1 a 6 & 207 & 58 \\
6 a 24 & & 28 \\
\hline Programas Sociais & 99 & 07 \\
Bolsa Família & 25 & 18 \\
Leite Pela Vida & 64 & 47 \\
Bolsa Família e Leite pela Vida & 169 & 24 \\
Não participam & & 61 \\
\hline Suplementação & 50 & 15 \\
Vitamina A & 126 & \\
Sulfato Ferroso & 31 & \\
Vitamina A e Sulfato Ferroso & & \\
\hline
\end{tabular}




\begin{tabular}{lcc}
\hline Condição de Habitação & & \\
Alugada & 212 & 59 \\
Cedida & 34 & 10 \\
Própria & 68 & 19 \\
Não relataram & 43 & 12 \\
\hline Ordem de filhos & & 52 \\
Primeiro & 184 & 31 \\
Segundo & 109 & 13 \\
Terceiro & 46 & 4 \\
Quarto/ mais & 18 & \\
\hline Classificação Econômica Brasil & & 8 \\
Classe A & 29 & 45 \\
Classe B & 161 & 47 \\
Classe C & 167 & \\
\hline
\end{tabular}

Fonte: Os autores (2014)

Quanto à alimentação infantil, observou-se que 140 (93\%) das crianças menores de seis meses haviam sido amamentadas no dia anterior à coleta de dados, 44 (29\%) haviam consumido fórmula infantil e $32(21 \%)$ haviam consumido água. Entre as crianças com mais de seis meses, 93 (45\%) consumiram leite materno no dia antecedente à pesquisa.

$\mathrm{Na}$ análise da alimentação complementar, investigou-se o consumo de alimentos fontes de ferro e vitamina $C$ entre as crianças com idade de 6 a 24 meses (Tabela 2). Em relação ao grupo das carnes, a carne cozida/moída era consumida por $80(38,2 \%)$ crianças pelo menos uma vez por dia. Observou-se que $147(70,6 \%)$ crianças nunca consumiam ou consumiam em baixa frequência as vísceras (fígado de boi/miúdos de galinha) e de forma moderada e frequente a carne de frango.

Entre os vegetais, verificou-se que a maioria não consumia os folhosos como alface, acelga, repolho, espinafre e couve. Entre aqueles com classificação de consumo frequente, a cenoura e a abóbora, 74 $(35 \%)$, foram os itens mais citados. Quanto aos demais vegetais, prevaleceu-se o consumo moderado (Tabela 2).

As frutas consumidas frequentemente pelas crianças foram banana, 87 (41,5\%); e laranja, 74 (35,5\%). Aproximadamente metade das crianças nunca consumiu melão, melancia, manga, morango, uva e goiaba. O uso de sucos naturais foi observado em 122 (58,5\%) crianças da população estudada, que os consumiam de modo frequente (Tabela 2).

O consumo de feijão esteve presente na alimentação de todas as crianças de forma moderada ou frequente (Tabela 2).

Tabela 2 - Porcentagem de frequência do consumo de frutas e vegetais, segundo o grupo de crianças que iniciaram alimentação complementar (n=209). Montes Claros, MG, Brasil, 2014. (continua)

\begin{tabular}{lccccccccc}
\hline & \multicolumn{1}{c}{ Frequência de consumo } \\
\hline Alimento & \multicolumn{2}{c}{ Nunca } & \multicolumn{2}{c}{ Baixa } & \multicolumn{2}{c}{ Moderada } & \multicolumn{2}{c}{ Frequente } \\
\hline Vegetais & $\mathbf{n}$ & $\%$ & $\mathbf{n}$ & $\%$ & $\mathbf{n}$ & $\%$ & $\mathbf{n}$ & $\%$ \\
Alface & 116 & 55,5 & 25 & 11,9 & 50 & 23,8 & 18 & 8,8 \\
Acelga/repolho/espinafre/couve manteiga & 145 & 69,5 & 0 & & 58 & 27,6 & 6 & 2,9 \\
Couve-flor & 166 & 79,4 & 12 & 5,9 & 25 & 11,8 & 6 & 2,9 \\
Beterraba & 63 & 30,1 & 6 & 2,9 & 105 & 50,3 & 35 & 16,7 \\
Cenoura & 43 & 20,5 & 6 & 2,9 & 86 & 41,3 & 74 & 35,3 \\
\hline
\end{tabular}


Cogitare Enferm. (23)2: e51908, 2018

\begin{tabular}{lcccccccc}
\hline Tomate & 86 & 41,2 & 6 & 2,9 & 55 & 26,5 & 61 & 29,4 \\
Abóbora & 18 & 8,8 & 12 & 5,9 & 105 & 50,1 & 74 & 35,2 \\
\hline Frutas & 12 & 5,9 & 0 & 0 & 110 & 52,6 & 87 & 41,5 \\
$\quad$ Banana & 18 & 8,8 & 12 & 5,9 & 104 & 49,8 & 74 & 35,5 \\
Laranja & 12 & 5,9 & 24 & 11,7 & 104 & 49,8 & 68 & 32,6 \\
Maça/pêra & 74 & 35,3 & 25 & 11,8 & 74 & 35,3 & 37 & 17,6 \\
Mamão & 85 & 40,8 & 37 & 17,7 & 74 & 35,6 & 12 & 5,9 \\
Melão/melancia & 91 & 43,7 & 50 & 23,8 & 43 & 20,7 & 25 & 11,8 \\
Manga & 86 & 41,2 & 74 & 35,3 & 31 & 14,7 & 18 & 8,8 \\
Morango & 92 & 44,1 & 74 & 35,3 & 37 & 17,7 & 6 & 2,9 \\
Uva & 110 & 52,9 & 62 & 29,5 & 31 & 14,7 & 6 & 2,9 \\
Goiaba & 12 & 5,9 & 12 & 5,9 & 62 & 29,7 & 122 & 58,5 \\
$\quad$ Suco de frutas natural & & & & & & & & \\
\hline Leguminosas & 0 & 0 & 0 & 0 & 43 & 20,6 & 166 & 79,4 \\
$\quad$ Feijão & & & & & & & & 0 \\
\hline Carnes e ovos & 86 & 41,2 & 61 & 29,4 & 61 & 29,4 & 0 & 0 \\
\hline Fígado de boi/miúdos de galinha & 43 & 20,6 & 12 & 5,9 & 74 & 35,3 & 80 & 38,2 \\
Carne cozida/carne moída & 80 & 38,2 & 25 & 11,8 & 74 & 35,3 & 31 & 14,7 \\
Bife & 49 & 23,5 & 12 & 5,9 & 117 & 55,9 & 31 & 14,7 \\
Frango cozido/assado/grelhado/frito & 153 & 73,5 & 31 & 14,7 & 25 & 11,8 & 0 & 0 \\
Peixe cozido/frito & 92 & 44,1 & 37 & 17,6 & 80 & 38,2 & 0 & 0 \\
Ovo frito/mexido/cozido & & & & & & & &
\end{tabular}

Fonte: Os autores (2014)

Na análise da suplementação de ferro e vitamina A, verificou-se que 127 (61\%) das crianças maiores de seis meses estavam recebendo a suplementação de ambos os micronutrientes, enquanto 31 (15\%) receberam apenas a vitamina A e 50 (24\%) receberam apenas o sulfato ferroso.

\section{DISCUSSÃO}

Este estudo avaliou o consumo de alimentos fontes de ferro e vitamina C entre crianças de até 24 meses de idade. Nos primeiros seis meses de vida, a prática do aleitamento materno deste estudo foi expressiva, o que constitui um fator protetor para a prevenção da anemia ferropriva ${ }^{(15)}$. Entres as crianças na faixa etária de 6 a 24 meses de idade houve baixa frequência de consumo de alimentos fontes de ferro e de alimentos facilitadores de sua absorção, considerado como fator de risco para a deficiência desse micronutriente no organismo ${ }^{(2)}$.

$\mathrm{Na}$ análise de pesquisas nacionais realizadas de 1975 a 2008 no Brasil, foi observado aumento expressivo na duração mediana da amamentação ${ }^{(16)}$. A adesão à amamentação nesse estudo foi similar ao encontrado em Diadema, SP, em que observou-se a prevalência de 80,9\% de aleitamento materno em crianças menores de 6 meses e de $44,4 \%$ em crianças de 6 a 23 meses ${ }^{(17)}$.

O consumo da fórmula infantil observado nessa pesquisa, em um terço das crianças, foi inferior a prevalência de aproximadamente $50 \%$ de uso no estudo supracitado ${ }^{(17)}$. Na ausência da amamentação exclusiva, o consumo da fórmula infantil deve ser a primeira opção de escolha na alimentação do lactente. O leite de vaca in natura é desaconselhado nessa fase, diante das inadequações nutricionais e do risco de anemia ferropriva ${ }^{(17)}$. Há que se considerar ainda que o consumo de água e fórmula infantil entre as crianças menores de seis meses pesquisadas pode refletir o possível comprometimento da prática do aleitamento materno exclusivo preconizado nessa faixa etária. 
A partir dos seis meses o consumo do leite materno foi observado em quase a metade das crianças pesquisadas, assim como reportados por outros autores ${ }^{(17)}$. Nessa fase, apesar do leite materno não atender às demandas nutricionais necessárias, é uma fonte complementar de ferro na alimentação infantil e deve ser consumido até os 24 meses de idade ${ }^{(9)}$.

A alimentação das crianças pesquisadas com idade de 6 a 24 meses foi deficiente de alimentos fontes de ferro e vitamina $C$. As práticas alimentares estão relacionadas com a presença de anemia ferropriva na infância. Nessa fase, a necessidade de ferro precisa ser suprida por meio da alimentação complementar variada e em quantidades adequadas que propicie o aporte apropriado desse nutriente ${ }^{(18)}$. A primeira infância é um período de maior vulnerabilidade para o desenvolvimento da anemia ferropriva, devido ser a etapa da vida em que há a maior taxa de crescimento e, por conseguinte, gera necessidades nutricionais aumentadas ${ }^{(1-3)}$.

O consumo de carnes nessa pesquisa apresentou frequências concordantes ao verificado em levantamento nacional com crianças na mesma faixa etária ${ }^{(3)}$. Esse resultado sugere que as crianças não consomem suficientemente fontes alimentares de ferro, já que as carnes são alimentos com elevada quantidade desse mineral ${ }^{(2)}$. O consumo inadequado de carnes nessa fase do ciclo de vida pode ser justificado pela dificuldade de aceitação em relação à sua consistência ou ainda pelo custo elevado(2). Ressalta-se que, além das carnes vermelhas, as vísceras são boas fontes de ferro ${ }^{(9)}$. Nesse estudo, a maior parte das crianças não tem o hábito de consumi-las. Na fase da introdução da alimentação complementar, as crianças refletem as práticas alimentares da família e, considerando que no país há um consumo de vísceras insuficiente na fase adulta, esse padrão pode refletir no hábito infanti ${ }^{(19)}$.

Em relação ao grupo dos vegetais, observou-se baixo consumo de folhosos. Esses achados estão em consonância com a realidade de crianças atendidas na Estratégia Saúde da Família no estado do Acre, em que 53,4\% das crianças de 6 a 24 meses não consumiram hortaliças nas refeições principais ${ }^{(20)}$. Esse resultado pode está relacionado com o fato de que a maior parte da população brasileira consome as hortaliças abaixo das recomendações nutricionais ${ }^{(19)}$. Há de se refletir que os vegetais folhosos verdes-escuros são fontes razoáveis de ferro na dieta ${ }^{(9)}$.

As frutas, fontes de vitamina $C$, são consideradas facilitadores da absorção de ferro e o seu consumo é indispensável ${ }^{(9)}$. Nesse estudo foi baixo o consumo diário de uma variedade de frutas nas faixas etárias de 6 a 24 meses, o que compromete a qualidade da dieta. A literatura evidencia dados mais críticos para essa população, em que $48,2 \%$ das crianças não ingerem nenhuma fruta ${ }^{(20)}$. Considerando que as práticas alimentares encontram-se vinculadas à disponibilidade de alimentos, às características culturais e à escolha dos mesmos, ${ }^{(21)}$ as frutas típicas da região nunca foram consumidas por parcela expressiva das crianças pesquisadas, o que também sugere exposição à hábitos alimentares familiares inadequados.

O consumo comum de feijão nas crianças desse estudo reflete o hábito alimentar da população brasileira $^{(21,22)}$ e constitui-se como um fator protetor para a anemia infantil ${ }^{(2)}$.

A suplementação de ferro e vitamina A, preconizada pelo Ministério da Saúde, mostrou-se insuficiente na população estudada. O uso de suplementos nutricionais de ferro nos dois primeiros anos de vida é a primeira e mais tradicional estratégia para a prevenção e o tratamento da anemia por deficiência de ferro preconizado no SUS, ${ }^{(9)}$ visto que as necessidades desse nutriente nessa faixa etária são grandes e difíceis de serem atingidas na dieta. Associado a essa suplementação, a vitamina A em níveis adequados no organismo podem contribuir para elevar o ferro orgânico na população ${ }^{(23)}$.

Assim como os dados obtidos nesse estudo, a Pesquisa Nacional de Saúde mostrou que a suplementação com sulfato ferroso foi referida para mais da metade das crianças com idade entre 6 e 23 meses, superior às pesquisas prévias realizadas no país. Esse aumento na frequência de crianças suplementadas pode ser explicado devido a melhorias no acesso ao suplemento mineral no âmbito da Atenção Primária à Saúde no Brasil( ${ }^{(3)}$.

A Estratégia Saúde da Família (ESF) tem papel importante na promoção de hábitos alimentares saudáveis na infância ${ }^{(18)}$. Percebe-se o êxito no incentivo ao aleitamento materno realizado pelas equipes de saúde da família, especialmente na atuação do enfermeiro ${ }^{(24)}$. Todavia, há a necessidade de melhorar as orientações sobre a alimentação complementar, principalmente no que diz respeito às fontes de ferro e vitamina C para a prevenção da anemia ferropriva ${ }^{(9)}$. 
Para tanto, são essenciais a realização de atividades educativas individuais ou coletivas sobre as práticas alimentares na prevenção de anemia ferropriva, durante as consultas de puericultura ou nos grupos educativos realizadas na APS potencialmente pelo profissional de enfermagem. É essencial que essas orientações educativas considerem o nível de escolaridade materna ou do responsável e a condição financeira da família - nesta pesquisa mostrou-se baixo - o que pode contribuir para que os conceitos aprendidos sejam aplicados na realidade em que estão inseridos. O enfermeiro precisa considerar o contexto sociocultural de cada nutriz para melhor adesão às recomendações oferecidas ${ }^{(24,25)}$.

A ESF, ao desenvolver ações em um território geograficamente conhecido, possibilita aos profissionais de saúde uma proximidade para conhecer seus contextos de vida, o que constitui um fator favorecedor para mudanças de prática. Ações em saúde que considerem o contexto em que as escolhas alimentares ocorrem, podem subsidiar propostas de intervenção efetivas para a promoção da alimentação saudável nesse grupo da população, sendo que o enfermeiro tem sido protagonista para o desenvolvimento dessas ações no âmbito da atenção primária ${ }^{(24,25)}$.

Esse estudo teve como limitação as informações do hábito alimentar basear-se no relato das mães ou responsáveis. No entanto, a literatura aponta que o questionário de frequência de consumo alimentar é um instrumento adequado para avaliar hábitos alimentares em estudos epidemiológicos.

Os resultados deste estudo podem subsidiar estratégias de redução e de combate à anemia, considerando que devem fundamentar-se em análise de dados locais ${ }^{(8)}$.

\section{CONCLUSÕES}

O consumo de alimentos fontes de ferro e vitamina $C$ das crianças de 0 a 24 meses na atenção primária à saúde é de baixa frequência, o que pode favorecer o desenvolvimento de anemia ferropriva nessa população.

Espera-se que este estudo contribua para a reflexão dos profissionais de saúde e gestores sobre a importância do incentivo ao consumo alimentar adequado na infância, no que tange às fontes de ferro e vitamina C para prevenção da anemia ferropriva. Com isso, remete-se, especialmente ao enfermeiro da APS, à prática de vigilância alimentar, com ênfase no consumo destes nutrientes. Além disso, medidas de promoção de saúde devem ser implementadas, envolvendo estratégias de educação em saúde, sobre esta temática durante as consultas de puericultura e grupos operativos que integre a família e a comunidade. Os resultados deste estudo podem subsidiar a implementação de políticas de saúde mais efetivas para a promoção de hábitos alimentares saudáveis e na prevenção de doenças e agravos nutricionais, de modo a promover o adequado crescimento e desenvolvimento infantil.

Sugerem-se a realização de pesquisas que investiguem de forma quantitativa o consumo de ferro e vitamina $\mathrm{C}$ e ainda a sua relação com o estado nutricional das crianças.

\section{REFERÊNCIAS}

1. Braga JAP, Vitalle MSS. Deficiência de ferro na criança. Rev. Bras. Hematol. Hemoter. [Internet] 2010;32(Suppl 2):3844 [acesso em 15 jan 2017]. Disponível: http://dx.doi.org/10.1590/S1516-84842010005000054.

2. Mello CS, Barros KV, de Morais MB. Alimentação do lactente e do pré-escolar brasileiro: revisão da literatura. J. Pediatr. [Internet] 2016;92(5):451-63 [acesso em 22 fev 2017]. Disponível: http://dx.doi.org/10.1016/j.jped.2016.02.013.

3. Jaime PC, de Frias PG, Monteiro HOC, Almeida PVB, Malta DC. Assistência em saúde e alimentação não saudável em crianças menores de dois anos: dados da Pesquisa Nacional de Saúde, Brasil, 2013. Rev. Bras. Saúde Matern. Infant. [Internet] 2016;16(2):159-67 [acesso em 05 mar 2017]. Disponível: http://dx.doi.org/10.1590/1806-93042016000200005. 
4. Carvalho CA, Fonsêca PCA, Priore SE, Franceschini SCC, Novaes JF. Consumo alimentar e adequação nutricional em crianças brasileiras: revisão sistemática. Rev. Paul. Pediatr. [Internet] 2015;33(2):212-21 [acesso em 12 fev 2017 ]. Disponível: http://dx.doi.org/10.1016/j.rpped.2015.03.002.

5. Allali S, Brousse V, Sacri AS, Chalumeau M, de Montalembert M. Anemia in children: prevalence, causes, diagnostic work-up, and long-term consequences. Expert. Rev. Hematol. [Internet] 2017;10(11):1023-28 [acesso em 16 fev 2018 ]. Disponível: http://doi.org/10.1080/17474086.2017.1354696.

6. McCarthy EK, ní Chaoimh C, Hourihane JO, Kenny LC, Irvine AD, Murray DM, et al. Iron intakes and status of 2-yearold children in the Cork BASELINE Birth Cohort Study. Maternal \& Child Nutrition. [Internet] 2017;13(3) [acesso em 16 fev 2018]. Disponível: https://doi.org/10.1111/mcn.12320.

7. Petry N, Olofin I, Hurrell RF, Boy E, Wirth JP, Moursi M, Donahue Angel M, et al. The Proportion of Anemia Associated with Iron Deficiency in Low, Medium, and High Human Development Index Countries: A Systematic Analysis of National Surveys. Nutrients. [Internet] 2016;8(11) [acesso em 16 fev 2018]. Disponível: https://doi.org/10.3390/nu8110693.

8. van derMerwe LF, Eussen SR. Iron status of young children in Europe. Am. J. Clin. Nutr. [Internet] 2017;106(Suppl6):1663S1671S [acesso em 16 fev 2018]. Disponível: https://doi.org/10.3945/ajcn.117.156018.

9. Ministério da Saúde (BR). Secretaria de Atenção à Saúde. Departamento de Atenção Básica. Programa Nacional de Suplementação de Ferro: Manual de condutas gerais. [Internet] Brasília: Ministério da Saúde; 2013 [acesso em 18 mar 2017]. Disponível: http://bvsms.saude.gov.br/bvs/publicacoes/manual_suplementacao_ferro_condutas_gerais.pdf.

10. Vieira RCS, Ferreira HS. Prevalência de anemia em crianças brasileiras, segundo diferentes cenários epidemiológicos. Rev. Nutr. [Internet] 2010;23(3):433-44 [acesso em 15 mar 2017]. Disponível: http://dx.doi.org/10.1590/S141552732010000300011.

11. de Oliveira TSC, da Silva MC, Santos JN, Rocha DS, Alves CRL, Capanema FD, et al. Anemia entre pré-escolares um problema de saúde pública em Belo Horizonte, Brasil. Ciênc. saúde coletiva. [Internet] 2014;19(1):59-66 [acesso em 03 mar 2017]. Disponível: http://dx.doi.org/10.1590/1413-81232014191.1927.

12. Zuffo CRK, Osório MM, Taconeli CA, Schmidt ST, da Silva BHC, Almeida CCB. Prevalence and risk factors of anemia in children. J. Pediatr. [Internet] 2016;92(4):353-60 [acesso em 20 fev 2017]. Disponível: http://dx.doi.org/10.1016/j. jped.2015.09.007.

13. Barbosa RMS, Peixoto NGA, Pereira AS, Vieira CBL, Soares EA, Lanzillotti HS. Estudo de prevalência de adequação de Ferro e Vitamina C em dietas infantis. Rev. Bras. Epidemiol. [Internet] 2014;17(2):543-56 [acesso em 04 abr 2017]. Disponível: http://dx.doi.org/10.1590/1809-4503201400020019ENG.

14. Ministério da Saúde (BR). Secretaria de Atenção à Saúde. Departamento de Atenção Básica. Protocolos do Sistema de Vigilância Alimentar e Nutricional - SISVAN na assistência à saúde. [Internet] Brasília: Ministério da Saúde; 2013. [acesso em 18 mar 2017]. Disponível: http://bvsms.saude.gov.br/bvs/publicacoes/protocolo_sistema_vigilancia_alimnetar.pdf.

15. Marques RFSV, Taddei JAAC, Konstantyner T, Marques ACV, Braga JAP. Correlação entre níveis de hemoglobina de mães e filhos em aleitamento materno exclusivo no primeiro semestre de vida. J. Pediatr. [Internet] 2016;92(5):479-85 [acesso em 06 fev 2017]. Disponível: http://dx.doi.org/10.1016/j.jped.2015.11.006.

16. Venancio SI, Saldiva SRDM, Monteiro CA. Tendência secular da amamentação no Brasil. Rev. Saúde Pública. [Internet] 2013;47(6):1205-8 [acesso em 10 fev 2017]. Disponível: http://dx.doi.org/10.1590/S0034-8910.2013047004676.

17. Coelho LC, Asakura L, Sachs A, Erbert I, Novaes CRL, Gimeno SGA. Sistema de Vigilância Alimentar e Nutricional/ SISVAN: conhecendo as práticas alimentares de crianças menores de 24 meses. Ciência \& Saúde Coletiva. [Internet] 2015;20(3):727-38 [acesso em 12 mar 2017]. Disponível: http://dx.doi.org/10.1590/1413-81232015203.15952014.

18. Oliveira CSM, Augusto RA, Muniz PT, da Silva SA, Cardoso MA. Anemia e deficiência de micronutrientes em lactentes atendidos em unidades básicas de saúde em Rio Branco, Acre, Brasil. Ciência \& Saúde Coletiva. [Internet] 2016;21(2):517-29 [acesso em 20 mar 2017]. Disponível: http://dx.doi.org/10.1590/1413-81232015212.19072014. 
19. Instituto Brasileiro de Geografia e Estatística (IBGE). Pesquisa de orçamentos familiares 2008-2009: análise do consumo alimentar pessoal no Brasil. [Internet] Rio de Janeiro: IBGE; 2011. [acesso em 12 fev 2017]. Disponível: http:// biblioteca.ibge.gov.br/visualizacao/livros/liv50063.pdf.

20. Garcia MT, Granado FS, Cardoso MA. Alimentação complementar e estado nutricional de crianças menores de dois anos atendidas no Programa Saúde da Família em Acrelândia, Acre, Amazônia Ocidental Brasileira. Cad. Saúde Pública. [Internet] 2011;27(2):305-16 [acesso em 25 mar 2017]. Disponível: http://dx.doi.org/10.1590/S0102$311 \times 2011000200012$.

21. Souza AM, Pereira RA, Yokoo EM, Levy RB, Sichieri R. Alimentos mais consumidos no Brasil: Inquérito Nacional de Alimentação 2008-2009. Rev. Saúde Pública. [Internet] 2013;47(1 Suppl):191S-9S [acesso em 17 fev 2017]. Disponível: http://dx.doi.org/10.1590/S0034-89102013000700005.

22. Malta DC, Andrade SSCA, Stopa SR, Pereira CA, Szwarcwald CL, da Silva Junior JB, et al. Estilos de vida da população brasileira: resultados da Pesquisa Nacional de Saúde, 2013. Epidemiol. Serv. Saúde. [Internet] 2015;24(2):217-26 [acesso em 10 fev 2017]. Disponível: http://www.scielo.br/scielo.php?pid=S2237-96222015000200217\&script=sci_ abstract\&tlng=pt.

23. Saraiva BCA, Soares MCC, dos Santos LC, Pereira SCL, Horta PM. Iron deficiency and anemia are associated with low retinol levels in children aged 1 to 5 years. J. Pediatr. [Internet] 2014;90(6):593-99 [acesso em 15 mar 2017]. Disponível: http://dx.doi.org/10.1016/j.jped.2014.03.003.

24. Fonseca-Machado MO, Haas VJ, Stefanello J, Nakano AMS, Gomes-Sponholz F. Aleitamento materno: conhecimento e prática. Rev. esc. enferm. USP. [Internet] 2012;46(4):809-15 [acesso em 14 fev 2017]. Disponível: http://dx.doi. org/10.1590/S0080-62342012000400004.

25. Campos AMS, Chaoul CO, Carmona EV, Higa R, do Vale IN. Prática de aleitamento materno exclusivo informado pela mãe e oferta de líquidos aos seus filhos. Rev. Latino-Am. Enfermagem. [Internet] 2015;23(2):283-90 [acesso em 23 jan 2017]. Disponível: http://dx.doi.org/10.1590/0104-1169.0141.2553. 